\title{
Comparing and modeling the spread of early farming across Europe
}

Marc Vander Linden ${ }^{1}$ and Fabio Silva 2,3

\begin{abstract}
We review modeling approaches and meta-analyses of the archaeology on the introduction of domesticated plants and animals across Europe. Variation in the rates of diffusion, human demography and farming systems suggest complex patterns of land-use and land-cover changes.
\end{abstract}

The European Early Neolithic is defined by the introduction and subsequent diffusion across Europe of numerous plants and animals originally domesticated in the Levant. We offer here an overview of the archaeological literature focusing on (1) measures of the rate of spread; (2) the demographic background; and (3) the variability of early farming systems. The research strategy benefiting from these various literature reviews aims to, first, analyze large-scale datasets (i.e. meta-analysis) to detect patterns in the archaeological record, and, second, identify the variables responsible for these regularities using models. Particular attention is paid to the interplay between climatic, environmental and human factors.

\section{A question of time, not speed}

Seminal work by Ammerman and CavalliSforza (1971) showed a correlation between the local date of introduction of farming across Europe, and the distance from the source of domesticates i.e. older sites in the Near East and gradually younger ones as the spread proceeded through Europe. They also calculated that this progression operated at a mean rate of $1.08 \mathrm{~km}$ per year, explained through a model of demic diffusion, wherein the next generation of farmers would move, on average, $25 \mathrm{~km}$ away from their birthplace. Ancient DNA studies have recently confirmed that the introduction of domesticates is indeed linked to the dispersal of a new human population, with a limited contribution of the last local foraging communities, though the picture changes regionally (Lazaridis et al. 2016). Several papers have since adjusted Ammerman and Cavalli-Sforza's original estimate, but also pointed to regional variation in this movement estimation. For instance, Bocquet-Appel et al. (2009) showed that, while an average rate of $1.09 \mathrm{~km}$ per year can be calculated, it masks considerable variation, concluding that the spread of early farming across Europe was a discontinuous process, with successive episodes of expansion and stasis. Figure 1 illustrates this spatio-temporal structure: early domesticates reached Greece by ca. 8600 ka BP. After a standstill of a few centuries, the spread of early farming occurred

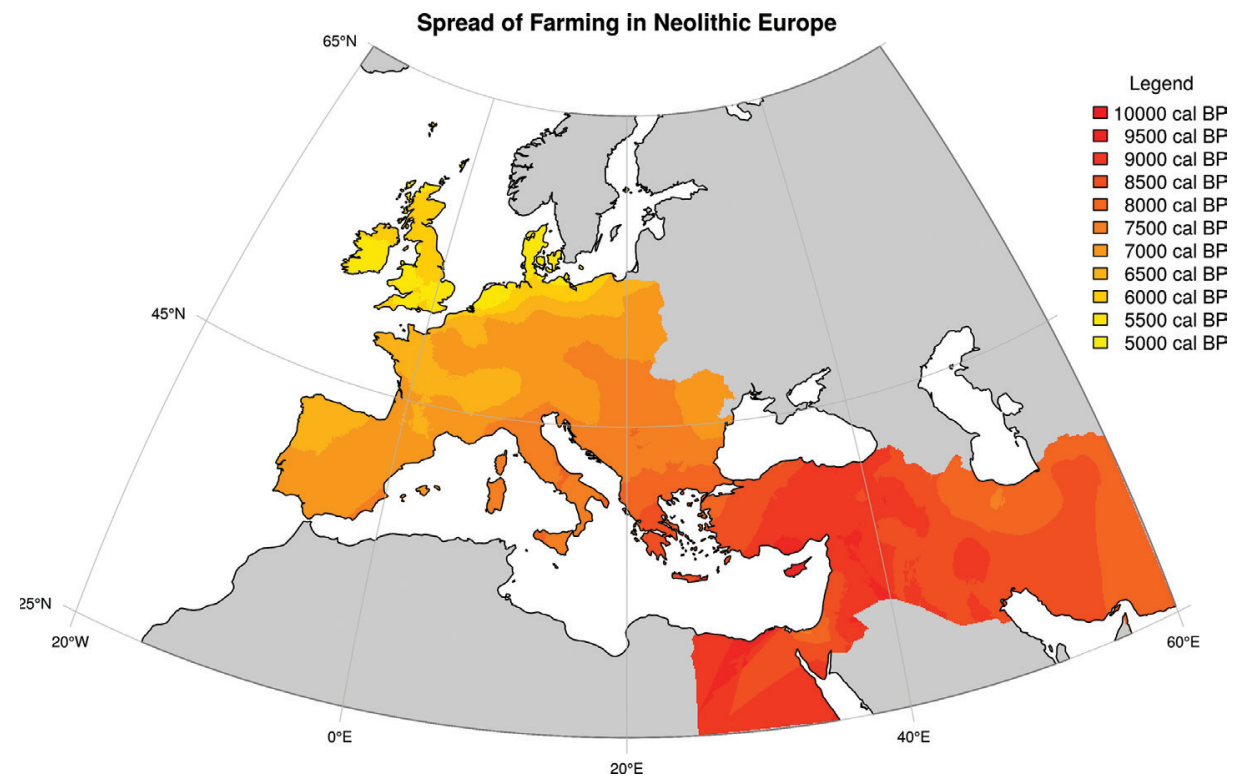

Figure 1: Geostatistical interpolation of radiocarbon dates related to the spread of early farming across the Near East and Europe. Note that the interpolation for Egypt in solely based on a few dates from the Sinai, and therefore should be considered with care for the rest of the region.

along two axes across Europe, firstly along the northern Mediterranean coast and reaching the Iberian Peninsula by the mid 7th millennium before present (7.5 ka BP) and, secondly, along the Rhine-Danube corridor up to the Paris basin by the early 7th millennium before present (7 ka BP). After a long standstill in central and parts of continental NW Europe, the spread resumed once again by the early 6th millennium before present (6 ka BP), when the expansion happened into southern Scandinavia, Britain and Ireland.

Several factors affect these rates of dispersal. For instance, rapid extreme climatic episodes linked to the $8.2 \mathrm{ka} \mathrm{BP}$ "cold event" shaped the spread of farming in the southern Balkans (Krauss et al., in press), and Warden et al. (2017) have argued for a relationship between climatic amelioration and the introduction of farming in southern Scandinavia. Another approach lies in comparing radiocarbon dates to computational simulations of the diffusion process and associated parameters, especially the mean spatial dispersal rate, and population growth rate. Such recent models incorporate important geographic features such as river valleys and coastlines and include the acceleration of the spread along them. These models also take into account the "slowing down" of dispersal related to high elevations and latitudes. The resulting simulations are shown to better match empirical data than simulations from earlier models (Silva and Steele 2014).

\section{Agriculture and demography}

The introduction of early farming in Europe is related to the inception of a new human population, and recent work points to the complex demographic history of Neolithic Europe. Fluctuations in population density have been inferred through statistical analyses of radiocarbon dates, assuming that the extent of past populations and their activities is reflected in the number of samples dated by archaeologists. Shennan et al. (2013) identified an apparent recurrent pattern of "boom-bust" (increases and decreases of the number of radiocarbon dates) in several regions of western Europe. The local introduction of farming corresponds to a rise in the radiocarbon record linked to demographic growth assumed from the 

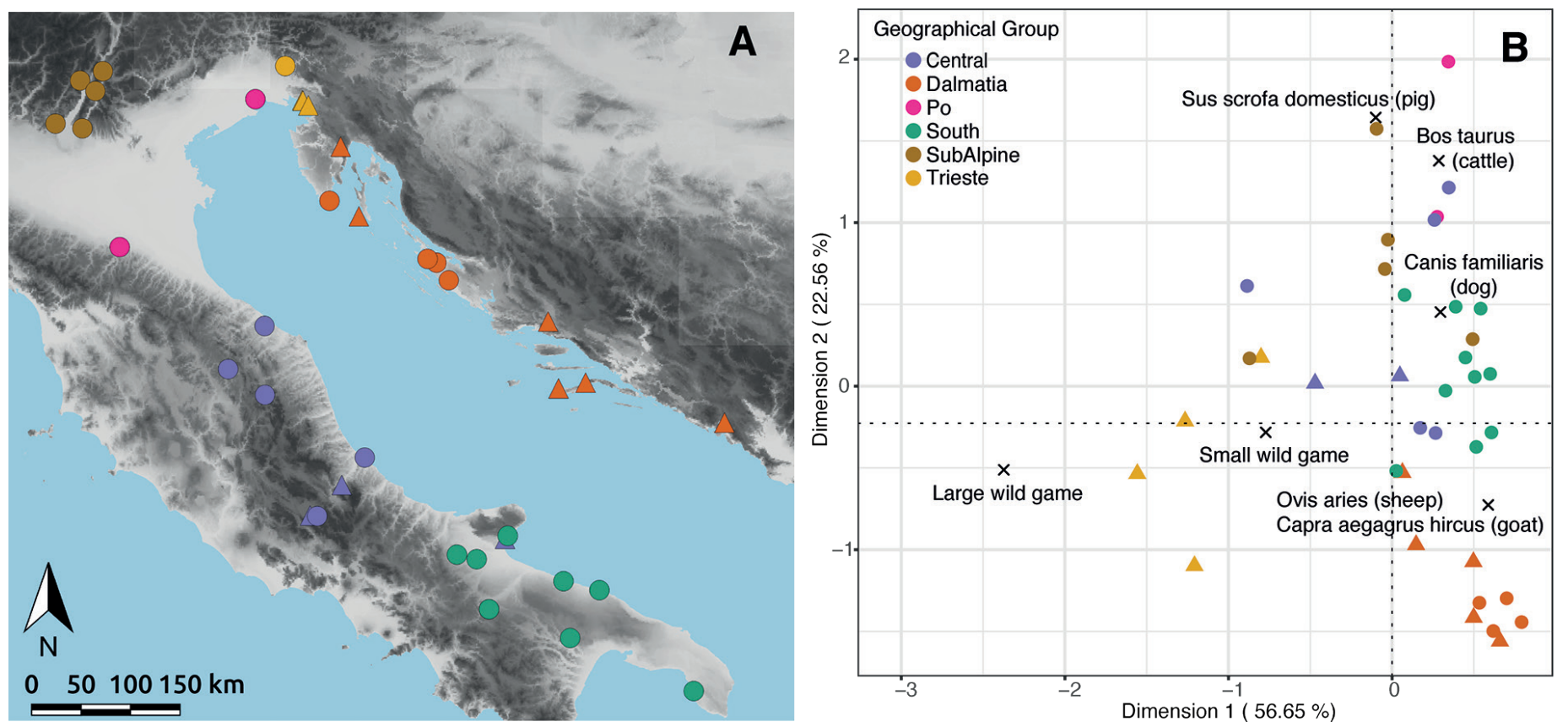

Figure 2: (A) Map of the earliest regional Neolithic zooarchaeological assemblages in the Adriatic basin. (B) Correspondence analysis for the sites on the map (circle: open air site; triangle: cave site).

introduction of farming; it is followed by a decline in the density of radiocarbon dates interpreted as a depletion of the population. In Britain and Ireland, fluctuations in the radiocarbon record match changes in vegetation cover, where the "boom" is related to deforestation, and the "bust" to woodland regeneration. This sequence occurs during worsening climatic conditions, and is associated with transformations in agricultural regimes, though the precise interplay between all factors is complex (Whitehouse et al. 2014; Woodbridge et al. 2014). It must be stressed that the identification, characterization and interpretation of these fluctuations remain debated. For instance, Silva and Vander Linden (2017) observed that the "bust" in one area sometimes matched the "boom" in the adjacent region during the spread, suggesting that the demographic depletion may also partly correspond to the outgoing migration of a fraction of the local population.

\section{Describing farming variability}

Less attention has been paid to landuse patterning in archaeobotanical and zooarchaeological assemblages, due to limited systematic sampling and reporting. Europe sees the introduction of cattle (Bos taurus), pigs (Sus scrofa domesticus), sheep (Ovis aries) and goats (Capra aegagrus hircus) and early Neolithic zooarchaeological assemblages present extensive variations in their respective proportions. Cattle and pigs dominate in central, northern and northwestern Europe, and sheep/goats in the Mediterranean (Manning et al. 2013). Multi-linear regression suggests that environmental factors account for 23-30\% of the variation in domesticated animals and cultural factors for a further $10 \%$, with the rest of the variation being left unexplained, at least at this scale (Manning et al. 2013). Indeed, these broad patterns mask extensive regional diversity. Figure 2 presents a correspondence analysis of the earliest regional Neolithic zooarchaeological assemblages across the Adriatic basin. Neolithic sites in southern Italy and Dalmatia are dominated by sheep and goats. Their dominance gradually decreases in central and northern Adriatic in favor of more water-demanding cattle and pigs, mirroring the gradient in precipitation observed across the Adriatic (Gaastra and Vander Linden, in press).

Existing meta-analyses point to a gradual loss of diversity in plant domesticates, especially as farming spreads from the Balkans into Central Europe during the late 7th to early 6th millennium before present (7-6 ka BP; Colledge et al. 2005). As changes in environmental factors alone cannot account for this pattern, two alternative hypotheses are considered: either this narrower crop package corresponds to a cultural preference by early farmers, or is the outcome of neutral drift, a stochastic process linked to what is being transmitted within a small population undergoing expansion. Competing agentbased models indicate that both hypotheses cannot be rejected, leaving open the interpretation of this pattern observable in archaeobotanical data (Conolly et al. 2008; Pérez-Losada and Fort 2011).

\section{Conclusion}

The diffusion of early farming across Europe corresponds to the introduction of several plant and animal domesticates, and of a new human population. This process lasted nearly three millennia and comprised cycles of expansion and stasis. Meta-analyses of archaeobotanical and zooarchaeological records demonstrate large variations in the types of cultivars and domesticates in use, shaped by a combination of environmental, climatic, and human factors, which remain difficult to disentangle. This variety of rates of diffusion, associated demographic signals, and crop-domesticated animal packages suggests likely significant differences in land use across the continent, and associated land-cover changes. Hopefully, future work will address the imbalance between the extensive efforts in analyzing and modeling radiocarbon dates, and the relative scarcity of similar approaches for in-depth analysis of plant and animal remains to improve the understanding of spatial and temporal patterning of animal and crop cultivation practices.

\section{AFFILIATIONS}

'Department of Archaeology, University of Cambridge, UK

${ }^{2}$ Catalan Institute of Human Paleoecology and Social Evolution, Tarragona, Spain

${ }^{3}$ Department of Historical Sciences, Rovira i Virgili University, Tarragona, Spain

\section{CONTACT}

Marc Vander Linden: mv297@cam.ac.uk

\section{REFERENCES}

Ammerman AJ, Cavalli-Sforza L-L (1971) Man NS 6: 674-688

Bocquet-Appel J-P et al. (2009) J Archaeol Sci 36: 807-820

Colledge S et al. (2005) Eur J Archaeol 8: 137-156

Conolly J et al. (2008) J Archaeol Sci 35: 2797-2804 Gaastra J, Vander Linden M (in press) Holocene Krauss $R$ et al. (in press) Quat Int

Lazaridis I et al. (2016) Nature 536: 419-424

Manning Ket al. (2013) Antiquity 87: 1046-1059

Pérez-Losada J, Fort J (2011) J Archaeol Sci 38: 1294-1299 Shennan S et al. (2013) Nat Commun 4: 2486 Silva F, Steele J (2014) J Archaeol Sci 52: 609-620 Silva F, Vander Linden M (2017) Sci Rep 7: 11985

\section{Warden L et al. (2017) Sci Rep 7: 15521}

Whitehouse N et al. (2014) J Archaeol Sci 51: 181-205 Woodbridge J et al. (2014) J Archaeol Sci 51: 216-224 